\title{
LAS TAC Y LOS RECURSOS PARA GENERAR APRENDIZAJE
}

LKT and the resources to generate learning

TAC e os recursos para gerar aprendizagem

\section{Manuel Ángel Velasco Rodríguez}

Colegio Santa Teresa (León), España. Teléfono: +34 600275 468. Correo electrónico: manuvelasco@hotmail.es

\section{Resumen}

En el contexto educativo las TAC (Tecnologías del Aprendizaje y el Conocimiento) tratan de reconducir las TIC (Tecnologías de la Información y Comunicación) hacia un uso más formativo y pedagógico. De este modo, las TAC van más allá de aprender a utilizar las TIC y nos permiten explorar estas herramientas tecnológicas al servicio del aprendizaje y de la adquisición de conocimiento. Por ello, el presente taller pretende centrarse en aquellos aspectos necesarios para introducir de forma efectiva las TAC en el proceso de enseñanza-aprendizaje: actualización continua de conocimientos y habilidades, competencia digital, nueva conceptualización de la enseñanza, generación de entornos virtuales de aprendizaje y transformación de los roles del profesorado y del alumnado. Se ofrecerá un variado repositorio de recursos TAC que nos permitan aprender más, aprender siempre y aprender para toda la vida. Para finalizar se compartirán usos reales de las TAC a través de propuestas didácticas adaptadas al aula y extrapolables a otros contextos.

Palabras clave: Buenas prácticas; TAC; Aprendizaje; recursos para generar aprendizaje; TIC

\begin{abstract}
In the educational context, the LKTs (Learning and Knowledge Technologies) seek to bring ICTs (Information and Communication Technologies) towards a more formative and pedagogical use. In this way, LKTs go beyond learning to use ICTs alone and are committed to exploring these technological tools in the service of learning and the acquisition of knowledge. For this reason, the present workshop aims to focus on those aspects necessary to effectively introduce LKTs into the teaching - learning process:
\end{abstract}


continuous updating of knowledge and skills, digital competence, new conceptualization of teaching, generation of virtual learning environments and Transformation of the roles of teachers and students. We will offer a varied repository of LKT resources that will allow us to learn more, always learn and learn for a lifetime. Finally, we will share real uses of LKTs through didactic proposals adapted to the classroom and extrapolated to other contexts.

Keywords: Good practices; LKT; Learning; Resources to generate learning; ICT

\section{Resumo}

No contexto educacional, as TAC (Tecnologias de aprendizagem e conhecimento) tentam redirecionar TIC (Informação e Comunicação) para uma utilização mais educativa e pedagógica. Assim, as TAC ir além de simplesmente aprender a utilizar as TIC e estão empenhados em explorar essas tecnologias ao serviço de ferramentas de aprendizagem e de aquisição de conhecimento. Portanto, este workshop pretende concentrar-se nos aspectos necessários para efetivamente introduzir o TAC no processo de ensino - aprendizagem: actualização contínua dos conhecimentos e habilidades, competência digital, nova concepção de ensino, geração de ambientes virtuais de aprendizagem e mudança de papéis de professores e alunos. uma riqueza de recursos TAC repositório que nos permitem aprender, aprender sempre e aprender para a vida será oferecido. Para terminar usos reais do TAC através de propostas educacionais adaptadas à sala de aula e extrapolados para outros contextos ação.

Palavras-chave: Boas práticas; TAC; aprendizagem; gerar recursos para a aprendizagem; TIC

\section{La necesidad de transformar los roles del profesorado y del alumnado como} punto de partida

El rol del profesorado se verá afectado con la introducción de las TAC en su práctica docente, ya que las estrategias implementadas en situaciones convencionales de enseñanza ya no son satisfactorias (Salinas, 2004). El cambio de un escenario tradicional del proceso de enseñanza-aprendizaje a un entorno tecnológico da lugar a una redefinición de sus tareas, donde las actitudes, la competencia y su formación muestran una influencia sustancial en el proceso de adopción de la tecnología en su 
práctica docente (Newhouse, 2002). Por lo tanto, el profesor “debe dejar de ser un instructor que domina los conocimientos, para convertirse en asesor, orientador, facilitador y mediador del proceso de enseñanza-aprendizaje” (Segura, Candioti, \& Medina, 2007, p. 6).

Por otro lado, el rol del alumnado también se ve modificado por las herramientas tecnológicas, pues la tecnología implica una mayor motivación en el aprendizaje (Area, 2010; Pedró, 2011), así como un cambio de actitudes que enriquece el aprendizaje, y que contribuye a ganar confianza en uno mismo (Pedró, 2011). Pero estos recursos educativos necesitan ser dominados para la construcción del conocimiento, con lo cual el alumnado precisa de nuevas destrezas para afrontar esta realidad (Salinas, 2010). Por tanto, el rol del alumno ya no es un mero reproductor de contenidos memorísticos sino que “debe llegar a ser un usuario inteligente y crítico de la información, para lo que precisa aprender a buscar, obtener, procesar y comunicar información y convertirla en conocimiento” (Segura, Candioti, \& Medina, 2007, p. 6).

Como vemos, estos cambios nos llevan claramente a un cambio de paradigma en la escuela y hacia una enseñanza de calidad, donde la motivación y la mejora de los resultados académicos estarán presentes (Pedró, 2011).

\section{Contextualización del taller: Todas las etapas educativas}

Los contenidos a abordar durante el desarrollo de este taller estarán centrados en todas las etapas educativas. Aunque la mayoría de los contenidos se tratarán de forma general, en ocasiones será necesario contextualizar el uso de ciertas estrategias y recursos para cada etapa educativa.

Asimismo, se pretende desarrollar el taller a través de una dinámica participativa que permita tener en cuenta las aportaciones de los asistentes con la intención de contextualizar cada una de las propuestas que se plantean a la diversidad de realidades educativas.

\section{Descripción del taller}

El taller se dividirá en dos partes. En un primer momento, se abordarán los aspectos necesarios para introducir de forma efectiva las TAC en el proceso de enseñanzaaprendizaje: actualización continua de conocimientos y habilidades, competencia 
digital, nueva conceptualización de la enseñanza, generación de entornos virtuales de aprendizaje y transformación de los roles del profesorado y del alumnado. También se descubrirán nuevos enfoques como las TEP (Tecnologías para el Empoderamiento y la participación) y las TPACK (Technological PedAgogical Content Knowledge).

En un segundo momento, cada profesor analizará el contexto educativo y tecnológico en el que desarrolla su función profesional (características del alumnado, capacidades y/o competencias a desarrollar en el alumnado, recursos disponibles, entre otros), para decidir entre algunos de las siguientes estrategias y recursos TAC que nos permiten adquirir conocimientos centrándose en los diferentes estilos de aprendizaje y teniendo muy presente que de nada sirven estas estrategias y recursos TAC si no los ponemos en las manos de los alumnos para que sean ellos los protagonistas y para que pasen de ser meros consumidores TIC a prosumers. Se presentarán un gran número de recursos TAC con los que los alumnos pueden crear diferentes contenidos y aprender. Ellos serán los protagonistas y responsables de su proceso de enseñanza-aprendizaje, siempre bajo la supervisión y acompañamiento del docente. Se profundizará más en los recursos que ofrecen un mayor potencial.

\section{Recursos TAC:}

Edición vídeo: Quik, Splice, Stupeflix y 123 apps. - Con estas herramientas los alumnos y docentes podrán crear y editar vídeos de una manera sencilla.

Vídeos interactivos: Vizia y EDpuzzle. - Estos recursos nos permiten convertir cualquier vídeo en una lección interactiva que captará la atención y interés del alumnado.

Audio: SoundCloud, Audacity, Peggo y Vocaroo. - Aplicaciones para crear archivos audio y poder compartirlos.

Imagen - infografías: Genially, Typorama, Fotojet y Piktochart. - El lenguaje visual es muy importante, estas herramientas permiten crear infografías de cualquier tipo.

Gestión del aula: iDoceo y Additio. - Aplicaciones para gestionar el día a día de un docente: listas, evaluación, rúbricas, banco de recursos, organización de grupos, etc. 
Gamificación: Cerebriti Edu, Quizizz, Trivinet, Jeopardy Rocks, Playbuzz, Triventy y Arcademic. - Recursos para gamificar el aula y dar vida a los contenidos curriculares.

Evaluación: Apester, Kahoot!, Google Forms y Quiz Revolution. - Herramientas que nos permiten comprobar los aprendizajes adquiridos por nuestros alumnos de una manera diferente.

Comunicación: Homeroom, Otter y Blogger. - Recursos para establecer una comunicación fluida entre todos los miembros de la comunidad educativa.

Presentanciones: Emaze, Genially, Powtoon, Sparkol y Haiku Deck. Herramientas para hacer presentaciones de una manera sencilla y con unos resultados atractivos y profesionales.

Generadores de actividades: Educaplay y Kubbu. - Aplicaciones para crear nuestras propias actividades interactivas de manera personalizada.

Trabajo colaborativo: Padlet, Team Maker, Teamweek, Meetingwords y Quip. Recursos que nos facilitan el trabajo colaborativo.

Buscadores y navegación segura: YouTube Kids y Kiddle. - Recursos para que los alumnos puedan buscar vídeos e información con total seguridad.

Derechos de autor: Pixabay, Iconicons, Skitterphoto, Sample Focus y pngimg. Herramientas para buscar imágenes, archivos audio, iconos, etc. sin derechos de autor para utilizar en nuestros proyectos.

Mapas mentales: Coggle y WiseMapping. - Aplicaciones para crear mapas mentales digitales.

Idiomas: Lyrics training, Locallingual y Forvo. - Recursos para aprender idiomas a través de audios reales y de situaciones interactivas.

Producciones: Pixton, Tiki toki y Storybird. - Herramientas para que nuestros alumnos creen cómics, líneas del tiempo o cuentos.

Otras utilidades: Small pdf, Refme, Videociborg y Jumpshare. - Aplicaciones que facilitan la tarea diaria de cualquier docente. 


\section{Conclusiones}

Es necesario que sepamos ver las nuevas posibilidades que las tecnologías abren a la educación cuando dejan de usarse como un elemento meramente instrumental cuyo objeto es hacer más eficiente el modelo educativo actual. Su nueva función pasa a ser posibilitar que "el contexto sociotecnológico genere un nuevo modelo de escuela que responda a las necesidades formativas de los ciudadanos” (Adell, 2013).

Si aprendemos a usar adecuadamente las TAC para motivar a los alumnos, potenciar su creatividad e incrementar sus habilidades multitarea, así como para aprovechar las sinergias entre profesores y estudiantes, conformaremos un aprendizaje aumentado. En este aprendizaje aumentado, los alumnos, de forma proactiva, autónoma, guiados por su curiosidad hacia un aprendizaje permanente, aprenden a sacar partido a la extraordinaria potencia de Internet como fuente de información, seleccionan y filtran recursos, se convierten en los protagonistas de las metodologías didácticas y reciben estímulos permanentes.

\section{Referencias}

Adell, J. (2013). La anatomía de los PLEs. En L. Castañeda y J. Adell (Eds.), Entornos Personales de Aprendizaje: Claves para el ecosistema educativo en red (pp. 1127). Alcoy: Marfil.

Area, M. (2010). El proceso de integración y uso pedagógico de las TIC en los centros educativos. Un estudio de casos. Revista Educación, 352, 77-97.

Salinas, J. (2004). Innovación docente y uso de las TIC en la enseñanza universitaria. Revista de Universidad y Sociedad del Conocimiento, 1(1). Recuperado de http://www.uoc.edu/rusc/dt/esp/salinas1104.pdf

Newhouse, C.P. (2002). The Impact of ICT on Learning and Teaching: A literature review for the Western Australian Department of Education. Recuperado de http://www.worldscientific.com/doi/abs/10.1142/S1793206808000483

Pedró, F. (2011). Tecnología y Escuela: lo que funciona y por qué. XXVI Semana Monográfica de la Educación. La Educación en la Sociedad Digital. Fundación Santillana. Recuperado de http://dide.minedu.gob.pe/handle/123456789/4870 
Segura, M., Candioti, C., \& Medina, C.J. (2007). Las TIC en la Educación: panorama internacional y situación española. XXII Semana Monográfica de la Educación, Fundación Santillana. Recuperado de http://www.oei.es/tic/DocumentoBasico.pdf 